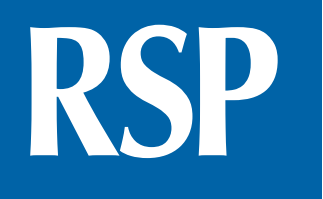

http://www.rsp.fsp.usp.br/
Revista de Saúde Pública

\title{
Violência contra a gestante e fatores associados no município de Governador Valadares
}

\author{
Érica Cesário Defilipo' iD, Paula Silva de Carvalho Chagas" iD, Luiz Cláudio Ribeiro"I' \\ I Universidade Federal de Juiz de Fora campus Governador Valadares. Instituto de Ciências da Vida. \\ Departamento de Fisioterapia. Governador Valadares, MG, Brasil \\ " Universidade Federal de Juiz de Fora. Faculdade de Fisioterapia. Programa de Pós-Graduação em Ciências da \\ Reabilitação e Desempenho Físico-funcional. Juiz de Fora, MG, Brasil \\ I"I Universidade Federal de Juiz de Fora. Departamento de Estatística. Programa de Pós-Graduação em Saúde \\ Coletiva. Juiz de Fora, MG, Brasil
}

Correspondência:

Érica Cesário Defilipo

Clínica Escola de Fisioterapia

Rua Leonardo Cristino, 3.400

35012-000 Governador Valadares,

MG, Brasil

E-mail: erica.defilipo@ufjf.br

Recebido: 26 mar 2020

Aprovado: 27 mai 2020

Como citar: Defilipo EC, Chagas PSC, Ribeiro LC. Violência contra a gestante e fatores associados no município de Governador Valadares. Rev Saude Publica. 2020;54:135.

Copyright: Este é um artigo de acesso aberto distribuído sob os termos da Licença de Atribuição Creative Commons, que permite uso irrestrito, distribuição e reprodução em qualquer meio, desde que o autor e a fonte originais sejam creditados.

\section{RESUMO}

OBJETIVO: Caracterizar a violência contra a mulher durante a gestação e verificar a associação com os fatores socioeconômicos, demográficos, obstétricos, comportamentais, de assistência à saúde e doenças na gestação.

MÉTODOS: Estudo transversal realizado com puérperas cujo parto ocorreu no Hospital Municipal de Governador Valadares, em Minas Gerais, no período de maio de 2017 a julho de 2018. A coleta de dados foi realizada por meio de entrevista, e informações complementares foram obtidas pela análise do cartão de pré-natal e prontuário. Para a análise dos dados, foi utilizada regressão logística.

RESULTADOS: Participaram do estudo 771 puérperas. Dessas, 62 (8,0\%) relataram ter sofrido violência física, psicológica ou sexual durante a gestação. As gestantes com maior chance de terem sofrido violência foram as dependentes de álcool $(\mathrm{RC}=4,97$; IC95\% 2,30-10,75; $\mathrm{p}<0,001)$, as que não realizaram o pré-natal $(\mathrm{RC}=3,88$; IC95\% 1,00-15,09; $\mathrm{p}=0,050)$, as que usaram os serviços de saúde de forma emergencial durante a gravidez $(\mathrm{RC}=2,47 ; \mathrm{IC} 95 \% 1,42-4,30 ; \mathrm{p}=0,001$ ) e que apresentaram diabetes gestacional $(\mathrm{RC}=2,59$; IC95\% 1,06-6,32; $\mathrm{p}=0,037)$ e doenças sexualmente transmissíveis ( $\mathrm{RC}=3,85$; IC95\% 1,41-10,50; $\mathrm{p}=0,009$ ).

CONCLUSÃO: A violência contra a gestante está associada a fatores comportamentais e relacionados à assistência à saúde e doenças na gestação. É imprescindível o reconhecimento dos fatores associados pelos profissionais de saúde, por meio de ações de rastreamento de situações de violência contra a mulher desde o início do pré-natal, a fim de possibilitar intervenção precoce.

DESCRITORES: Gestantes. Violência contra a Mulher. Fatores de Risco. Fatores Socioeconômicos. Estudos Transversais. 


\section{INTRODUÇÃO}

A violência contra a mulher é reconhecida como grave problema de saúde pública ${ }^{1}$, sendo definida como qualquer ato de violência de gênero que possa resultar em danos físicos, sexuais ou psicológicos ou sofrimento às mulheres². De acordo com a Organização Mundial de Saúde, cerca de uma em cada três mulheres no mundo já sofreram violência em algum momento da vida, em especial física, sexual ou ambas². Tal situação agrava-se quando se refere à mulher em um momento de grande fragilidade física e emocional como a gestação, pois apresenta uma ameaça para a mulher e para o feto, exigindo maior atenção dos serviços de saúde ${ }^{1,3}$.

A prevalência de violência contra a mulher durante a gestação varia entre diferentes comunidades, regiões e países ${ }^{4}$. A maioria dos países apresenta prevalências de violência na gestação entre $2 \%$ e 13,5\%, com maior predomínio nos países da África e da América Latina ${ }^{5}$. No Brasil, a proporção de mulheres que relataram violência por parceiro íntimo variou de cerca de 14 a $17 \%^{6}$, com prevalência de $8 \%$ nas gestantes 7 . A violência contra a mulher, em qualquer fase da vida, aumentou consideravelmente nos últimos anos no país, ocorrendo principalmente no Sudeste, Sul e Centro-Oeste ${ }^{8}$. No município de Governador Valadares, em Minas Gerais, a taxa de violência contra a mulher foi de $8,8 \%$ no ano de 2017, com maior quantitativo de vítimas de violência física, seguida da violência psicológica ${ }^{9}$, não havendo dados sobre a prevalência de violência contra a gestante no município.

Os efeitos maternos e neonatais da violência na gestação são considerados preveníveis. Para a gestante há maior risco de desenvolver depressão, ganho de peso insuficiente na gestação, dificuldade de realizar pré-natal de forma adequada e morte materna ${ }^{10}$. Para o feto, estudos demonstram maior risco de baixo peso ao nascer, prematuridade, alterações comportamentais e até morte neonatal ${ }^{3,10,11}$.

Na gravidez existem mais oportunidades para a triagem e intervenção precoce durante o pré-natal de rotina ou tratamento hospitalar, quando necessário ${ }^{10}$. No entanto, é preciso primeiramente identificar as gestantes em risco e também aquelas que sofrem violência, visto que esse assunto raramente é reportado pelas vítimas, sendo necessário o reconhecimento dos fatores associados pelos profissionais de saúde para possível intervenção precoce.

Este estudo objetivou caracterizar a violência contra a mulher durante a gestação e verificar a associação com os fatores socioeconômicos, demográficos, obstétricos, comportamentais, de assistência à saúde e doenças na gestação.

\section{MÉTODOS}

Estudo transversal, realizado com puérperas cujo parto ocorreu no período de maio de 2017 a julho de 2018 no Hospital Municipal de Governador Valadares, em Minas Gerais, escolhido por atender ao Sistema Único de Saúde e por ser considerado referência para a região do Vale do Rio Doce. Foram incluídas no estudo todas as puérperas participantes da pesquisa "Fatores associados à prematuridade e ao baixo peso ao nascer em Governador Valadares, Minas Gerais: estudo caso-controle"12, no qual foram considerados casos os nascidos vivos prematuros (idade gestacional inferior a 37 semanas) e também os nascidos vivos a termo (idade gestacional igual ou superior a 37 semanas e inferior a 42 semanas) com baixo peso ao nascer (inferior a 2.500 gramas), enquanto os controles foram os nascidos vivos a termo com peso adequado ao nascer (igual ou superior a 2.500 gramas), pareados pelo sexo e data de nascimento, sendo selecionados dois controles para cada caso. Foram excluídos do estudo os nascidos vivos com malformações congênitas, síndromes genéticas, doenças progressivas e lesões do sistema nervoso, diagnosticadas ou suspeitas ao nascimento. O estudo foi aprovado pelo Comitê de Ética e Pesquisa em Seres Humanos (CAAE: 61055716.4.0000.5147), sendo conduzido dentro dos padrões éticos exigidos. 
A coleta de dados foi realizada por meio de entrevista com as puérperas, ainda no período de internação hospitalar, em um prazo de 24 a 48 horas após o parto, e informações complementares foram obtidas por meio da análise do cartão de pré-natal e do prontuário da puérpera. Os dados foram coletados por pesquisadores previamente treinados. Todas as participantes assinaram o termo de consentimento livre e esclarecido

Foi considerada como variável dependente ter sofrido violência física, psicológica ou sexual na gravidez. Durante a entrevista, era perguntado à puérpera: "Você sofreu violência física, sexual ou psicológica durante sua gestação? Ou seja, alguém te ameaçou, agrediu, bateu, abusou sexualmente de você, te humilhou ou falou algo que você não gostou, controlou seu comportamento ou te deixou com medo?" No caso de resposta afirmativa, também foi questionado qual o tipo de violência sofrida (física, psicológica ou sexual).

As variáveis independentes incluídas na análise foram divididas em cinco blocos: 1) fatores socioeconômicos e demográficos; 2) fatores obstétricos; 3) fatores comportamentais; 4) fatores relacionados à assistência à saúde da gestante; e 5) doenças na gestação. As variáveis estudadas em cada bloco foram descritas de acordo com o modelo explicativo apresentado na Figura 1, na qual consta a forma de categorização de cada variável. A detecção do uso de

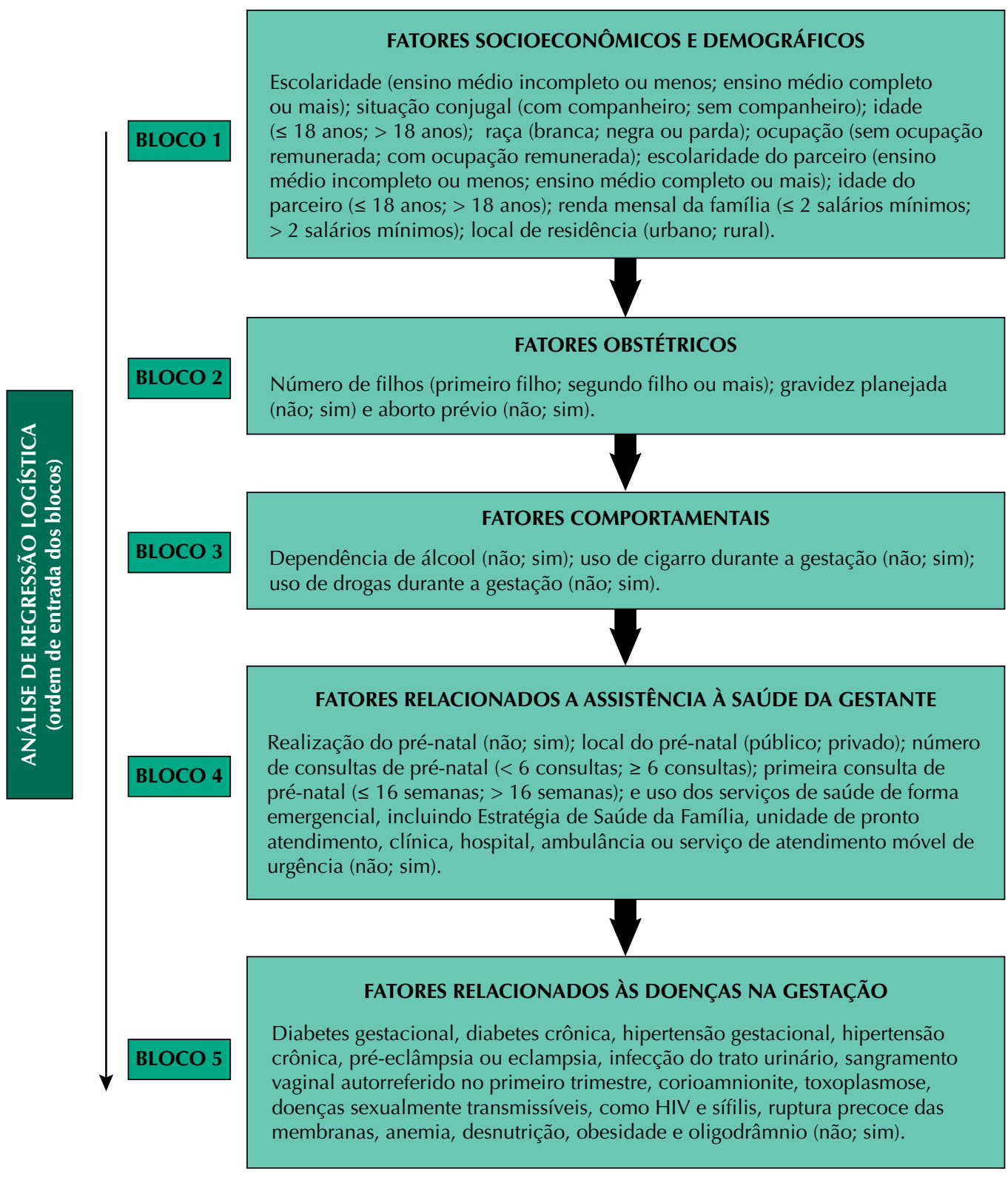

Figura. Modelo explicativo das variáveis independentes divididas em blocos e ordem de entrada dos fatores na análise de regressão logística. 
álcool foi realizada por meio do instrumento CAGE (cutdown, annoyed, guilty e eye-opener) ${ }^{13}$, escolhido por ser um instrumento de fácil aplicação, simples e com validade para uso no Brasil. Foram classificadas como dependentes do uso de álcool as mulheres que apresentaram uma resposta afirmativa ou mais. A categorização das variáveis "número de consultas de

Tabela 1. Frequência e número de gestantes expostas ou não à violência, razão de chances, intervalo de confiança de $95 \%$ e valor de $p$ dos fatores socioeconômicos e demográficos (bloco1) e obstétricos (bloco 2).

\begin{tabular}{|c|c|c|c|c|c|c|c|c|c|}
\hline \multirow{2}{*}{ Variáveis } & \multicolumn{2}{|c|}{$\begin{array}{l}\text { Frequência } \\
(\mathrm{n}=771)\end{array}$} & \multicolumn{2}{|c|}{$\begin{array}{c}\text { Expostas } \\
\text { à violência }\end{array}$} & \multicolumn{2}{|c|}{$\begin{array}{l}\text { Não expostas à } \\
\text { violência }\end{array}$} & \multirow{2}{*}{ RC } & \multirow{2}{*}{ IC95\% } & \multirow{2}{*}{$\mathbf{p}$} \\
\hline & $\mathbf{n}$ & $\%$ & $\mathbf{n}$ & $\%$ & $\mathrm{n}$ & $\%$ & & & \\
\hline
\end{tabular}

\section{Escolaridade}

EM incompleto ou menos

EM completo ou mais

Idade

$\leq 18$ anos

$>18$ anos

Raça

Branca

Negra ou parda

Situação conjugal

Com companheiro

Sem companheiro

Ocupação

Sem ocupação remunerada

Com ocupação remunerada

\section{Escolaridade do companheiro}

EM incompleto ou menos

EM completo ou mais

\section{Idade do companheiro}

$$
\leq 18 \text { anos }
$$

$>18$ anos

\section{Renda familiar}

$$
\begin{aligned}
& \leq 2 \text { salários mínimos } \\
& >2 \text { salários mínimos }
\end{aligned}
$$

\section{Local de residência}

Urbano

Rural

(1)

393

$51,0 \quad 39$

$62,9 \quad 354$

378

49,0

$\begin{array}{cll}99 & 12,8 & 12 \\ 672 & 87,2 & 50\end{array}$

37,1

355

$\begin{array}{cc}49,9 & \text { Ref } \\ 50,1 & 0,59\end{array}$

$0,34-1,01$

$0,063^{\text {b }}$

111

$\begin{array}{llllllll}111 & 14,4 & 7 & 11,3 & 104 & 14,7 & \text { Ref } & \\ 660 & 85,6 & 55 & 88,7 & 605 & 85,3 & 1,35 & 0,60-3,05\end{array}$

\begin{tabular}{|c|c|c|c|c|c|c|c|c|c|}
\hline \multicolumn{10}{|c|}{ BLOCO 2} \\
\hline \multicolumn{10}{|l|}{ Número de filhos } \\
\hline Primeiro filho & 378 & 49,0 & 33 & 53,2 & 345 & 48,7 & Ref & & \\
\hline Segundo filho ou mais & 393 & 51,0 & 29 & 46,8 & 364 & 51,3 & 0,83 & $0,50-1,40$ & 0,510 \\
\hline \multicolumn{10}{|l|}{ Gravidez planejada } \\
\hline Não & 429 & 55,6 & 33 & 53,2 & 396 & 55.9 & Ref & & \\
\hline Sim & 342 & 44,4 & 29 & 46,8 & 313 & 44,1 & 1,11 & $0,66-1,87$ & 0,690 \\
\hline \multicolumn{10}{|l|}{ Aborto prévio } \\
\hline Não & 640 & 83,0 & 47 & 75,8 & 593 & 83,6 & Ref & & \\
\hline Sim & 131 & 17,0 & 15 & 24,2 & 116 & 16,4 & 1,63 & $0,88-3,02$ & $0,115^{b}$ \\
\hline
\end{tabular}

$\begin{array}{lrrrrrrrr}613 & 79,5 & 43 & 69,4 & 570 & 80,4 & \text { Ref } & & \\ 158 & 20,5 & 19 & 30,6 & 139 & 19,6 & 1,81 & 1,02-3,21 & 0,039^{\mathrm{b}} \\ & & & & & & & & \\ 503 & 65,2 & 44 & 71,0 & 459 & 64,7 & \operatorname{Ref} & & \\ 268 & 34,8 & 18 & 29,0 & 250 & 35,3 & 0,75 & 0,43-1,33 & 0,404\end{array}$

$\begin{array}{lllllllll}403 & 58,2 & 26 & 51,0 & 377 & 58,8 & \text { Ref } & & \\ 289 & 41,8 & 25 & 49,0 & 264 & 41,2 & 1,37 & 0,78-2,43 & 0,275\end{array}$

EM: ensino médio; RC: razão de chances; IC95\%: intervalo de confiança de 95\%; Ref: categoria de referência

a Algumas puérperas não souberam ou não aceitaram informar os dados do companheiro, como escolaridade $(n=79)$ e idade $(n=22)$, além da renda familiar $(n=33)$, portanto esses dados foram considerados ausentes na análise.

${ }^{\mathrm{b}} \mathrm{p}<0,20$. 
pré-natal" e "primeira consulta de pré-natal" foi definida com base no preconizado pelo Ministério da Saúde, que determina o início da assistência pré-natal até a décima sexta semana de gestação e mínimo de seis consultas ${ }^{14}$.

Para verificar as associações das variáveis independentes com a violência contra a mulher durante a gestação, foi realizada regressão logística. Os fatores associados que, na análise bivariada, apresentaram p-valor inferior a 0,20 foram considerados elegíveis para compor os modelos multivariados. Foi realizada análise multivariada das variáveis de cada bloco separadamente, retirando as variáveis que perderam a significância. Em seguida, as variáveis anteriormente selecionadas, que apresentaram p-valor inferior a 0,05 , foram submetidas a nova análise multivariada, seguindo a ordem de entrada dos blocos: primeiro as variáveis do bloco 1 , seguidas das variáveis do bloco 2, bloco 3, bloco 4 e bloco 5 (Figura 1).

\section{RESULTADOS}

Das 771 puérperas participantes, $62(8,0 \%)$ relataram ter sofrido violência durante a gestação. Dessas, 49 (79,0\%) relataram violência psicológica, 11 (17,8\%) violência física e $2(3,2 \%)$ relataram ter sofrido violência sexual. Entre as gestantes que foram expostas à

Tabela 2. Frequência e número de gestantes expostas ou não à violência, razão de chances, intervalo de confiança $95 \%$ e valor de p dos fatores comportamentais (bloco 3) e fatores relacionados à assistência à saúde da gestante (bloco 4).

\begin{tabular}{|c|c|c|c|c|c|c|c|c|c|}
\hline \multirow{2}{*}{ Variáveis } & \multicolumn{2}{|c|}{$\begin{array}{l}\text { Frequência } \\
(\mathrm{n}=771)\end{array}$} & \multicolumn{2}{|c|}{$\begin{array}{l}\text { Expostas à } \\
\text { violência }\end{array}$} & \multicolumn{2}{|c|}{$\begin{array}{c}\text { Não expostas à } \\
\text { violência }\end{array}$} & \multirow{2}{*}{ RC } & \multirow{2}{*}{ IC95\% } & \multirow{2}{*}{ p } \\
\hline & $\mathrm{n}$ & $\%$ & $\mathbf{n}$ & $\%$ & $\mathrm{n}$ & $\%$ & & & \\
\hline
\end{tabular}

\section{BLOCO 3}

\begin{tabular}{|c|c|c|c|c|c|c|c|c|c|}
\hline \multicolumn{10}{|c|}{ Dependência de álcool } \\
\hline Não & 726 & 94,2 & 50 & 80,6 & 676 & 95,3 & Ref & & \\
\hline Sim & 45 & 5,8 & 12 & 19,4 & 33 & 4,7 & 4,92 & $2,39-10,11$ & $<0,001^{*}$ \\
\hline \multicolumn{10}{|l|}{ Uso de cigarro } \\
\hline Não & 710 & 92,1 & 52 & 83,9 & 658 & 92,8 & Ref & & \\
\hline Sim & 61 & 7,9 & 10 & 16,1 & 51 & 7,2 & 2,48 & $1,19-5,17$ & $0,012^{*}$ \\
\hline \multicolumn{10}{|l|}{ Uso de drogas } \\
\hline Não & 763 & 99,0 & 60 & 96,8 & 703 & 99,2 & Ref & & \\
\hline Sim & 8 & 1,0 & 2 & 3,2 & 6 & 0,8 & 3,91 & $0,78-19,77$ & $0,076^{*}$ \\
\hline \multicolumn{10}{|c|}{ BLOCO 4} \\
\hline \multicolumn{10}{|c|}{ Realização do pré-natal } \\
\hline Não & 12 & 1,6 & 4 & 6,5 & 8 & 1,1 & Ref & & \\
\hline Sim & 759 & 98,4 & 58 & 93,5 & 701 & 98,9 & 0,17 & $0,05-0,57$ & $0,001^{*}$ \\
\hline \multicolumn{10}{|l|}{ Local do pré-natal } \\
\hline Público & 658 & 86,7 & 52 & 89,7 & 606 & 86,4 & Ref & & \\
\hline Privado & 101 & 13,3 & 6 & 10,3 & 95 & 13,6 & 0,74 & $0,31-1,76$ & 0,687 \\
\hline \multicolumn{10}{|c|}{ Número de consultas de pré-natal } \\
\hline$<6$ consultas & 190 & 24,9 & 23 & 37,1 & 167 & 23,9 & Ref & & \\
\hline$\geq 6$ consultas & 572 & 75,1 & 39 & 62,9 & 533 & 76,1 & 0,53 & $0,31-0,92$ & $0,021^{*}$ \\
\hline \multicolumn{10}{|c|}{ Primeira consulta de pré-natal } \\
\hline$\leq 16$ semanas & 636 & 84,8 & 51 & 87,9 & 585 & 84,5 & Ref & & \\
\hline$>16$ semanas & 114 & 15,2 & 7 & 12,1 & 107 & 15,5 & 0,75 & $0,33-1,70$ & 0,572 \\
\hline \multicolumn{10}{|c|}{ Uso emergencial dos serviços de saúde } \\
\hline Não & 490 & 63,6 & 28 & 45,2 & 462 & 65,2 & Ref & & \\
\hline Sim & 281 & 36,4 & 34 & 54,8 & 247 & 34,8 & 2,27 & $1,35-3,83$ & $0,002^{*}$ \\
\hline
\end{tabular}

RC: razão de chances; IC95\%: intervalo de confiança de 95\%; Ref: categoria de referência $* p<0,20$. 
violência, houve predomínio de mulheres com baixa escolaridade (ensino médio incompleto ou menos), negras ou pardas, sem ocupação remunerada, com companheiro e com renda inferior a dois salários mínimos.

$\mathrm{Na}$ análise bivariada dos fatores socioeconômicos e demográficos, apresentaram $\mathrm{p}<0,20$ as seguintes variáveis: escolaridade ( $p=0,063)$, idade $(p=0,110)$ e situação conjugal $(p=0,039)$. Dos fatores obstétricos, apenas a variável ter sofrido aborto prévio $(\mathrm{p}=0,115)$ apresentou p-valor inferior a 0,20 (Tabela 1$)$.

Com relação aos fatores comportamentais, todas as variáveis analisadas apresentaram associação com a violência contra a gestante: dependência de álcool ( $p<0,001)$, uso de cigarro ( $p=0,012)$ e uso de drogas ( $p=0,076)$. Já no que diz respeito aos fatores relacionados

Tabela 3. Frequência e número de gestantes expostas ou não à violência, razão de chances, intervalo de confiança 95\% e valor de p das doenças na gestação (bloco 5).

\begin{tabular}{|c|c|c|c|c|c|c|c|c|c|}
\hline \multirow{2}{*}{ Variáveis } & \multicolumn{2}{|c|}{$\begin{array}{c}\text { Frequência } \\
(\mathrm{n}=771)\end{array}$} & \multicolumn{2}{|c|}{$\begin{array}{c}\text { Expostas à } \\
\text { violência }\end{array}$} & \multicolumn{2}{|c|}{$\begin{array}{c}\text { Não expostas à } \\
\text { violência }\end{array}$} & \multirow{2}{*}{ RC } & \multirow{2}{*}{ IC95\% } & \multirow{2}{*}{$\mathbf{p}$} \\
\hline & $\mathbf{n}$ & $\%$ & $\mathbf{n}$ & $\%$ & $\mathbf{n}$ & $\%$ & & & \\
\hline \multicolumn{10}{|c|}{ Diabetes gestacional } \\
\hline Não & 732 & 94,9 & 55 & 88,7 & 677 & 95,5 & Ref & & \\
\hline Sim & 39 & 5,1 & 7 & 11,3 & 32 & 4,5 & 2,69 & $1,14-6,38$ & $0,020^{*}$ \\
\hline \multicolumn{10}{|c|}{ Hipertensão gestacional } \\
\hline Não & 671 & 87,0 & 53 & 85,5 & 618 & 87,2 & Ref & & \\
\hline Sim & 100 & 13,0 & 9 & 14,5 & 91 & 12,8 & 1,15 & $0,55-2,42$ & 0,706 \\
\hline \multicolumn{10}{|c|}{ Hipertensão crônica } \\
\hline Não & 752 & 97,5 & 61 & 98,4 & 691 & 97,5 & Ref & & \\
\hline Sim & 19 & 2,5 & 1 & 1,6 & 18 & 2,5 & 0,63 & $0,08-4,80$ & 1,000 \\
\hline \multicolumn{10}{|c|}{ Pré-eclâmpsia ou eclâmpsia } \\
\hline Não & 734 & 95,2 & 61 & 98,4 & 673 & 94,9 & Ref & & \\
\hline Sim & 37 & 4,8 & 1 & 1,6 & 36 & 5,1 & 0,31 & $0,04-2,27$ & 0,352 \\
\hline \multicolumn{10}{|c|}{ Infecção do trato urinário } \\
\hline Não & 474 & 61,5 & 31 & 50,0 & 443 & 62,5 & Ref & & \\
\hline Sim & 297 & 38,5 & 31 & 50,0 & 266 & 37,5 & 1,67 & $0,99-2,80$ & $0,053 *$ \\
\hline \multicolumn{10}{|c|}{ Sangramento vaginal no primeiro trimestre } \\
\hline Não & 650 & 84,3 & 44 & 71,0 & 606 & 85,5 & Ref & & \\
\hline Sim & 121 & 15,7 & 18 & 29,0 & 103 & 14,5 & 2,41 & $1,34-4,33$ & $0,003^{*}$ \\
\hline \multicolumn{10}{|c|}{ Oligodrâmnio } \\
\hline Não & 731 & 94,8 & 57 & 91,9 & 674 & 95,1 & Ref & & \\
\hline Sim & 40 & 5,2 & 5 & 8,1 & 35 & 4,9 & 1,69 & $0,64-4,48$ & 0,287 \\
\hline \multicolumn{10}{|c|}{ Ruptura prematura da membrana } \\
\hline Não & 741 & 96,1 & 61 & 98,4 & 680 & 95,9 & Ref & & \\
\hline Sim & 30 & 3,9 & 1 & 1,6 & 29 & 4,1 & 0,38 & $0,05-2,87$ & 0,503 \\
\hline \multicolumn{10}{|l|}{ Anemia } \\
\hline Não & 555 & 72,0 & 40 & 64,5 & 515 & 72,6 & Ref & & \\
\hline Sim & 216 & 28,0 & 22 & 35,5 & 194 & 27,4 & 1,46 & $0,85-2,52$ & $0,172^{*}$ \\
\hline \multicolumn{10}{|c|}{ DST (HIV ou sífilis) } \\
\hline Não & 747 & 96,9 & 56 & 90,3 & 691 & 97,5 & Ref & & \\
\hline Sim & 24 & 3,1 & 6 & 9,7 & 18 & 2,5 & 4,11 & $1,57-10,78$ & $0,002 *$ \\
\hline
\end{tabular}

DST: doenças sexualmente transmissíveis; RC: razão de chances; IC95\%: intervalo de confiança de 95\%; Ref: categoria de referência $* \mathrm{p}<0,20$ 
à assistência à saúde da gestante, apresentaram p-valor inferior a 0,20, na análise bivariada, as variáveis: realização de pré-natal $(\mathrm{p}=0,001)$, número de consultas $(\mathrm{p}=0,021)$ e uso emergencial dos serviços de saúde $(p=0,002)$, como se observa na Tabela 2 .

Dentre as doenças na gestação, foram selecionadas as variáveis: diabetes gestacional $(\mathrm{p}=0,020)$, infecção do trato urinário $(\mathrm{p}=0,053)$, sangramento vaginal no primeiro trimestre $(p=0,003)$, anemia $(p=0,172)$ e doenças sexualmente transmissíveis (DST) $(p=0,002)$, conforme a Tabela 3.

As variáveis selecionadas pela análise bivariada foram introduzidas em um modelo de regressão logística com análise por blocos. Na análise das variáveis do bloco 1 , a variável situação conjugal manteve associação significativa com a violência contra a gestante $(\mathrm{p}=0,041)$. No bloco 2 , a variável ter sofrido aborto prévio perdeu significância. Já no bloco 3 , a variável dependência de álcool apresentou associação significativa $(p<0,001)$. No bloco 4 , as variáveis que permaneceram no modelo foram: realização do pré-natal $(\mathrm{p}=0,001)$ e uso emergencial dos serviços de saúde ( $\mathrm{p}=0,001)$. Por fim, no bloco 5 , as variáveis que mantiveram associação significativa com a violência contra a gestante foram: diabetes gestacional $(p=0,030)$, sangramento vaginal no primeiro trimestre $(p=0,006)$ e DST $(\mathrm{p}=0,014)$. Na análise de regressão logística foram introduzidas, primeiramente, as variáveis selecionadas dos blocos 1 e 3, situação conjugal e dependência de álcool, sendo mantida no modelo apenas a variável dependência de álcool. Em seguida, foram introduzidas as variáveis do bloco 4, realização do pré-natal e uso emergencial dos serviços de saúde, e ambas foram mantidas no modelo. Por fim, foram acrescentadas as variáveis do bloco 5 , diabetes gestacional, DSTs e sangramento vaginal no primeiro trimestre, e a última foi retirada da análise por não apresentar associação significativa.

A Tabela 4 apresenta o resultado do modelo final da regressão logística, que indica que as variáveis dependência de álcool ( $p<0,001)$, uso emergencial dos serviços de saúde $(p=0,001)$, diabetes gestacional $(p=0,037)$ e DST $(p=0,009)$ apresentaram associação significativa com a violência contra a gestante. A variável realização de pré-natal apresentou p-valor bem próximo de ser significativo, sendo mantida no modelo para discussão $(p=0,050)$.

Tabela 4. Resultado final da regressão logística dos fatores associados à violência contra a mulher na gestação.

\begin{tabular}{|c|c|c|c|c|}
\hline Blocos & Variáveis & RC & IC95\% & p \\
\hline \multirow{3}{*}{ Bloco 3} & Dependência de álcool & & & \\
\hline & Não & Ref & & \\
\hline & $\operatorname{Sim}$ & 4,97 & $2,30-10,75$ & $<0,001^{*}$ \\
\hline \multirow{6}{*}{ Bloco 4} & Realização do pré-natal & & & \\
\hline & Sim & Ref & & \\
\hline & Não & 3,88 & $1,00-15,09$ & 0,050 \\
\hline & Uso de serviços de saúde emergencial & & & \\
\hline & Não & Ref & & \\
\hline & $\operatorname{Sim}$ & 2,47 & $1,42-4,30$ & $0,001^{*}$ \\
\hline \multirow{6}{*}{ Bloco 5} & Diabetes gestacional & & & \\
\hline & Não & Ref & & \\
\hline & $\operatorname{Sim}$ & 2,59 & $1,06-6,32$ & $0,037^{*}$ \\
\hline & Doenças sexualmente transmissíveis & & & \\
\hline & Não & Ref & & \\
\hline & Sim & 3,85 & $1,41-10,50$ & $0,009^{*}$ \\
\hline
\end{tabular}

RC: razão de chances; IC95\%: intervalo de confiança de 95\%; Ref: categoria de referência $* p<0,05$ 


\section{DISCUSSÃO}

A violência contra a mulher durante a gestação é motivo de grande preocupação de saúde global, visto que não apenas uma, mas duas vidas estão em risco ${ }^{3}$. Neste estudo, 8,0\% das puérperas entrevistadas relataram ter sofrido violência durante a gestação. Tal fato é preocupante e reforça a necessidade de atenção redobrada dos serviços de saúde por meio de ações preventivas e de rastreamento de situações de violência contra a mulher desde o início do pré-natal ${ }^{1,3}$.

Não foi observada associação entre violência contra a gestante e fatores socioeconômicos e demográficos. Da mesma forma, em um estudo brasileiro que analisou os efeitos do nível socioeconômico sobre a violência contra a mulher grávida, esse fator não esteve associado à violência física, psicológica ou sexual, tendo afetado gestantes de diferentes níveis socioeconômicos ${ }^{15}$. Por outro lado, outro estudo brasileiro observou que a baixa escolaridade aumentou em $1,5 \mathrm{vez}$ a chance de violência psicológica e quase dobrou a chance de violência física e sexual ${ }^{16}$. As gestantes com baixa escolaridade e renda relatam mais episódios de violência, o que demonstra a necessidade de identificação dessas mulheres que podem estar em risco $^{15}$.

As gestantes dependentes de álcool apresentaram maior chance de terem sido expostas à violência durante a gestação ( $\mathrm{RC}=4,97$; IC95\% 2,30-10,75; $\mathrm{p}<0,001)$. Vale ressaltar que a dependência de álcool neste estudo foi avaliada por meio de um instrumento padronizado para rastreio do uso de álcool. O uso frequente de álcool e a violência contra a mulher estão interligados, porém a natureza dessa associação é complexa, visto que o uso de álcool pode ser tanto a causa quanto a consequência da violência ${ }^{17}$. As mulheres podem utilizar a bebida para lidar com a violência, ao mesmo tempo que o fato de consumi-la pode resultar em violência, nos casos em que os parceiros não aceitam que a mulher consuma bebida alcóolica ${ }^{17}$. Dessa forma, a relação do uso de álcool e violência pode ser bidirecional. O consumo de bebida alcoólica está relacionado à menor união, harmonia e organização no ambiente familiar, assim como aos altos níveis de violência doméstica ${ }^{18}$. Tal situação torna-se ainda mais preocupante quando nos referimos ao consumo de bebida alcóolica durante a gestação. $\mathrm{O}$ uso de álcool pela gestante contribui para o ganho de peso gestacional insuficiente, maior utilização de outras drogas e menor comparecimento às consultas de pré-natal, além das repercussões diretas ao feto e recém-nascido, como maior risco de malformações, aborto espontâneo, prematuridade, baixo peso ao nascer, asfixia, mortalidade perinatal e síndrome do alcoolismo fetal ${ }^{19}$.

Experiências de violência durante a gestação estão associadas a comportamentos ou atitudes específicas, como o cuidado pré-natal inadequado ou início do acompanhamento $\operatorname{tardio}^{10,20}$. No presente estudo, as gestantes que não realizaram o pré-natal apresentaram 3,8 vezes mais chances de terem sofrido violência. Provavelmente devido ao pequeno número de puérperas que não realizaram o pré-natal $(\mathrm{n}=12)$, o intervalo de confiança foi amplo (IC95\% 1,00-15,09) e o p-valor foi ligeiramente superior ao valor de referência $(p=0,050)$. No entanto, como esse resultado apresentou associação bem próxima de ser significativa, merece ser discutido em estudos futuros, a fim de que os profissionais de saúde se atentem para esse fator. Audi et al. ${ }^{15}$, em estudo para identificar os fatores associados à violência contra gestantes acompanhadas em unidades de atenção primária à saúde no município de Campinas, São Paulo, observaram que a dificuldade em comparecer às consultas de pré-natal foi associada à violência física e sexual $(\mathrm{RC}=2,31 ; \mathrm{IC} 95 \% 1,18-4,51 ; \mathrm{p}=0,014)$. Outro estudo brasileiro, realizado no Rio de Janeiro, identificou que mulheres vítimas de violência buscam pela assistência pré-natal tardiamente, e as que relataram ter sido vítimas de abuso físico durante a gestação possuíam 2,2 vezes mais chance de apresentar uma assistência pré-natal inadequada do que aquelas sem história de violência ${ }^{21}$.

Mulheres expostas à violência sofrem constrangimentos de várias ordens, como ciúmes e ameaças, que resultam em restrição de sua liberdade ${ }^{15}$, podendo também ser desencorajadas pelos parceiros a realizarem o acompanhamento pré-natal ${ }^{22}$. Tal fato pode justificar a não 
realização ou não comparecimento em todas as consultas agendadas, conforme pode ser observado neste estudo, no qual 6,5\% das mulheres expostas à violência não realizaram o pré-natal e 37,1\% compareceram a menos de seis consultas. A facilidade de acesso à assistência pré-natal e o desenvolvimento de uma relação de confiança entre paciente e profissional de saúde são os primeiros passos para abordar o problema da violência na gravidez. Intervenções individualizadas e programas de visitas domiciliares direcionadas às gestantes que não comparecem às consultas agendadas podem ter resultados promissores ${ }^{9}$.

As gestantes que utilizaram os serviços de saúde de forma emergencial, incluindo consultas não agendadas na Estratégia Saúde da Família ou clínicas, uso de ambulância ou serviço de atendimento móvel de urgência, unidades de pronto atendimento e necessidade de internação em hospital, apresentaram maior chance de terem sido expostas à violência $(\mathrm{RC}=2,47 ; \mathrm{IC} 95 \%$ 1,42-4,30; $p=0,001)$. De acordo com a literatura, essa procura por serviços de saúde ocorre devido às consequências do elevado nível de estresse sofrido pela exposição à violência ou devido às lesões causadas pelas possíveis agressões físicas, ou ainda pelo trauma sexual ou infecções resultantes da violência sexual, o que pode acarretar complicações na gestação ${ }^{23}$.

Muitas mulheres, quando procuram o serviço de saúde para cuidar de lesões causadas por violência, relutam em revelar a fonte real da lesão, atribuindo-a a alguma outra causa, e a maioria dos serviços não coletam maiores informações ${ }^{4}$. As novas diretrizes da Organização Mundial da Saúde enfatizam a urgente necessidade de integrar essas questões nos currículos de graduação de todos os cursos da área da saúde, bem como treinar as equipes dos diversos serviços de saúde para entender a relação entre violência e problemas de saúde das mulheres e para intervir de forma adequada ${ }^{4}$.

A violência contra a mulher por parceiro íntimo é um importante contribuinte para a vulnerabilidade das mulheres às DST. Neste estudo, as mulheres com DST apresentaram maior chance de terem sido expostas à violência $(\mathrm{RC}=3,85$; IC95\% 1,41-10,50; $\mathrm{p}=0,009)$. Os mecanismos relacionados ao aumento dessa vulnerabilidade incluem a infecção direta por meio de relações sexuais forçadas, visto que mulheres em relacionamentos violentos podem ter controle limitado sobre o momento ou as circunstâncias da relação sexual e pouca capacidade de negociar o uso de preservativo ${ }^{4,24}$.

Um estudo de coorte realizado no sul da África encontrou que a desigualdade de gênero nos relacionamentos e a violência por parceiro íntimo aumentam o risco de infecção por HIV $(\mathrm{RC}=1,51 ; \mathrm{IC} 95 \% 1,04-2,21 ; \mathrm{p}=0,032)^{25}$. A história de violência por parceiro íntimo também apresentou associação significativa com o teste de sífilis positivo (RC = 1,61; IC95\% 1,24-2,08; $\mathrm{p}<0,01)$ em gestantes bolivianas, sugerindo que essa doença pode ser uma importante consequência negativa da violência para a saúde da mulher e da criança ${ }^{26}$. Outro estudo realizado na Índia, com o objetivo de descrever os fatores associados à incidência de DST, verificou que a incidência foi mais alta entre as mulheres casadas e expostas à violência sexual $^{27}$. As políticas, intervenções e programas de prevenção das DST devem abordar também esse importante fator de risco ${ }^{25}$.

Além das DST, as gestantes que apresentaram diabetes gestacional tiveram maior chance de terem sido expostas à violência $(\mathrm{RC}=2,59$; IC95\% 1,06-6,32; $\mathrm{p}=0,037)$. A diabetes mellitus gestacional representa o problema metabólico mais comum na gestação ${ }^{28}$, sendo que os fatores psicossociais e depressão podem contribuir para o seu desenvolvimento ${ }^{29}$. A violência física e psicológica está associada ao maior risco de diabetes tipo $2^{30}$, que por sua vez tem forte correlação com a diabetes gestacional, visto que os riscos metabólicos podem surgir primeiro na gravidez ${ }^{29}$. Além disso, mulheres com histórias de violência estão mais propensas à obesidade, um dos fatores de risco mais importante para a diabetes gestacional. Outro possível mecanismo biológico que explica essa associação é que a violência aumenta os níveis de hormônios do estresse que podem desencadear resistência à insulina ${ }^{29}$. Assim, os profissionais de saúde devem estar mais alertas para a identificação das mulheres com risco aumentado para desenvolver diabetes gestacional, que em alguns casos pode ser prevenível. 
Este estudo apresenta como limitação o fato de não ter sido utilizado nenhum instrumento padronizado para avaliar a violência contra mulher, sendo realizadas apenas perguntas diretas às puérperas sobre essa questão. $\mathrm{O}$ uso de um instrumento padronizado poderia detectar um maior número de puérperas que foram expostas à violência durante a gestação, mas isso não prejudicou os resultados. Neste estudo, 8,0\% das puérperas entrevistadas relataram ter sofrido violência durante a gestação, número semelhante às taxas encontradas no Brasil ${ }^{6}$. Este estudo não abordou apenas a violência por parceiro íntimo, assim como muitos estudos, mas a violência psicológica, física e sexual praticada por diferentes pessoas no ambiente familiar e na comunidade, incluindo também o parceiro íntimo. Outra limitação deste estudo refere-se à relação de causalidade reversa inerente aos estudos transversais.

A violência contra a mulher é um problema de saúde pública com proporção epidêmica, que permeia todo o mundo, colocando a saúde das mulheres em risco, limitando sua participação na sociedade e causando grande sofrimento humano ${ }^{4}$. O governo brasileiro, apesar de algumas iniciativas como a Lei Maria da Penha, deve progredir em termos de legislação e planos de ação para combater esse problema crescente ${ }^{7}$. É preciso ampliar o debate acerca da violência contra a mulher, em especial as gestantes, dentro dos serviços de saúde, promovendo a visibilidade dos agravos e dialogando sobre a emancipação de gênero e o empoderamento das mulheres. Programas efetivos para identificar as vítimas de violência contra a mulher e intervir ainda durante a gestação são fundamentais, especialmente na atenção primáriaa ${ }^{15,19}$.

\section{REFERÊNCIAS}

1. Baird K. Women's lived experience of domestic violence during pregnancy (1). Pract Midwife. 2015;18(3):27-31.

2. World Health Organization. Violence against women. Geneva: WHO; 2019 [citado 20 out 2019 ]. Disponível em: https://www.who.int/news-room/fact-sheets/detail/violence-against-women

3. Donovan BM, Spracklen CN, Schweizer ML, Ryckman KK, Saftlas AF. Intimate partner violence during pregnancy and the risk for adverse infant outcomes: a systematic review and meta-analysis. BJOG. 2016;123(8):1289-99. https://doi.org/10.1111/1471-0528.13928

4. World Health Organization, Department of Reproductive Health and Research; London School of Hygiene and Tropical Medicine; South African Medical Research Council. Global and regional estimates of violence against women: prevalence and health effects of intimate partner violence and non-partner sexual violence. Geneva: WHO; 2013 [citado 20 out 2019]. Disponível em: https://www.who.int/reproductivehealth/publications/ violence/9789241564625/en/

5. Drevies KM, Kishor S, Johnson H, Stöckl H, Bacchus LJ, Garcia-Moreno C, et al. Intimate partner violence during pregnancy: analysis of prevalence data from 19 countries. Reprod Health Matters. 2010;18(36):158-70. https://doi.org/10.1016/S0968-8080(10)36533-5

6. Bott S, Guedes A, Ruiz-Celis AP, Mendoza JA. Intimate partner violence in the Americas: a systematic review and reanalysis of national prevalence estimates. Rev Panam Salud Publica. 2019;43:e26. https://doi.org/10.26633/RPSP.2019.26

7. García-Moreno C, Jansen HAFM, Ellsberg M, Heise L, Watts C. WHO Multi-country Study on Women's Health and Domestic Violence against Women: initial results on prevalence, health outcomes and women's responses. Geneva: World Health Organization; 2005 [citado 20 out 2019]. Disponível em: https://www.who.int/reproductivehealth/publications/ violence/24159358X/en/

8. Rodrigues NCP, O'Dwyer G, Andrade MKN, Flynn MB, Monteiro DLM, Lino VTS. The increase in domestic violence in Brazil from 2009-2014. Cienc Saude Coletiva. 2017;22(9):2873-80. https://doi.org/10.1590/1413-81232017229.09902016

9. Governo do Estado de Minas Gerais, Sistema Integrado de Defesa Social, Centro Integrado de Informações de Defesa Social. Diagnóstico de violência doméstica e familiar nas regiões integradas de segurança pública de Minas Gerais: Registros Tentados e Consumados. Belo Horizonte, MG; 2018 [citado 20 out 2019]. Disponível em: http://www.seguranca.mg.gov.br/ images/2020/Maio/Diagnosticos/final_Diagnstico\%20violncia\%20domstica\%202015\%20a\%20 2017\%20-\%20MG\%20e\%20RISPs.pdf 
10. Alhusen JL, Ray E, Sharps P, Bullock L. Intimate partner violence during pregnancy: maternal and neonatal outcomes. J Women's Health (Larchmt). 2015;24(1):100-6. https://doi.org/10.1089/jwh.2014.4872

11. Berhanie E, Gebregziabher D, Berihu H, Gerezgiher A, Kidane G. Intimate partner violence during pregnancy and adverse birth outcomes: a case-control study. Reprod Health. 2019;16:22. https://doi.org/10.1186/s12978-019-0670-4

12. Defilipo EC. Fatores associados à prematuridade e ao baixo peso ao nascer em Governador Valadares, Minas Gerais: estudo caso controle [tese]. Juiz de Fora, MG: Faculdade de Medicina da Universidade Federal de Juiz de Fora; 2019.

13. Mayfield D, McLeod G, Hall P. The CAGE Questionnaire: validation of a new alcoholism screening instrument. Am J Psychiatry. 1974;131(10):1121-3. https://doi.org/10.1176/ajp.131.10.1121

14. Ministério da Saúde (BR), Secretaria Executiva. Programa Humanização do Parto: humanização no pré-natal e nascimento. Brasília, DF; 2002 [citado 20 out 2019]. Disponível em: http://bvsms.saude.gov.br/bvs/publicacoes/parto.pdf

15. Ribeiro MRC, Silva AAM, Britto e Alves MTSS, Batista RFL, Ribeiro CCC, Schraiber LB, et al. Effects of socioeconomic status and social support on violence against pregnant women: a structural equation modeling analysis. PLoS One. 2017;12(1):e0170469. https://doi.org/10.1371/journal.pone.0170469

16. Audi CAF, Segall-Corrêa AM, Santiago SM, Andrade MGG, Pérez-Escamilla R. Violência doméstica na gravidez : prevalência e fatores associados. Rev Saude Publica. 2008;42(5):877-85. https://doi.org/10.1590/S0034-89102008005000041

17. Devries KM, Child JC, Bacchus LJ, Mak J, Falder G, Graham K, et al. Intimate partner violence victimization and alcohol consumption in women: a systematic review and meta-analysis. Addiction. 2014;109(3):379-91. https://doi.org/10.1111/add.12393

18. Okada MM, Hoga LAK, Borges ALV, Albuquerque RS, Belli MA. Domestic violence against pregnant women. Acta Paul Enferm. 2015;28(3):270-4. https://doi.org/10.1590/1982-0194201500045

19. Moraes $\mathrm{CL}$, Reichenheim ME. Rastreamento de uso de álcool por gestantes de serviços públicos de saúde do Rio de Janeiro. Rev Saude Pública. 2007;41(5):695-703. https://doi.org/10.1590/S0034-89102007000500002

20. Shrestha M, Shrestha S, Shrestha B. Domestic violence among antenatal attendees in a Kathmandu hospital and its associated factors: a cross-sectional study. BMC Pregnancy Childbirth. 2016;16(1):360. https://doi.org/10.1186/s12884-016-1166-7

21. Moraes $\mathrm{CL}$, Arana FDN, Reichenheim ME. Violência física entre parceiros íntimos na gestação como fator de risco para a má qualidade do pré-natal. Rev Saude Publica. 2010;44(4):667-76. https://doi.org/10.1590/S0034-89102010000400010

22. Stöckl H, Watts C, Kilonzo Mbwambo JK. Physical violence by a partner during pregnancy in Tanzania: prevalence and risk factors. Reprod Health Matters. 2010;18(36):171-80. https://doi.org/10.1016/S0968-8080(10)36525-6

23. Coker AL, Sanderson M, Dong B. Partner violence during pregnancy and risk of adverse pregnancy outcomes. Paediatr Perinat Epidemiol. 2004;18(4):260-9. https://doi.org/10.1111/j.1365-3016.2004.00569.x

24. Chambliss LR. Intimate partner violence and its implication for pregnancy. Clin Obstet Gynecol. 2008;51(2):385-97. https://doi.org/10.1097/GRF.0b013e31816f29ce

25. Jewkes RK, Dunkle K, Nduna M, Shai N. Intimate partner violence, relationship power inequity, and incidence of HIV infection in young women in South Africa: a cohort study. Lancet. 2010;376(9734):41-8. https://doi.org/10.1016/S0140-6736(10)60548-X

26. Díáz-Olavarrieta C, Wilson KS, García SG, Revollo R, Richmond K, Paz F, et al. The co-occurrence of intimate partner violence and syphilis among pregnant women in Bolivia. J Women's Health (Larchmt). 2009;18(12):2077-86. https://doi.org/10.1089/jwh.2008.1258

27. Weiss HA, Patel V, West B, Peeling RW, Kirkwood BR, Mabey D. Spousal sexual violence and poverty are risk factors for sexually transmitted infections in women: a longitudinal study of women in Goa, India. Sex Transm Infect. 2008;84(2):133-9. https://doi.org/10.1136/sti.2007.026039 
28. Wilson BL, Dyer JM, Latendresse G, Wong B, Baksh L. Exploring the psychosocial predictors of gestational diabetes and birth weight. J Obstet Gynecol Neonatal Nurs. 2015;44(6):760-71. https://doi.org/10.1111/1552-6909.12754

29. Mason SM, Tobias DK, Clark CJ, Zhang C, Hu FB, Rich-Edwards JW. Abuse in Childhood or Adolescence and Gestational Diabetes: a retrospective cohort study. Am J Prev Med. 2016;50(4):436-44. https://doi.org/10.1016/j.amepre.2015.08.033

30. Mason SM, Wright RJ, Hibert EN, Spiegelman D, Jun HJ, Hu FB, et al. Intimate partner violence and incidence of type 2 diabetes in women. Diabetes Care. 2013;36(5):1159-65. https://doi.org/10.2337/dc12-1082

Financiamento: Coordenação de Aperfeiçoamento de Pessoal de Nível Superior - Brasil (Capes - Código de Financiamento 001). Programa PROQUALI da Universidade Federal de Juiz de Fora (bolsa de doutorado concedida à primeira autora durante a realização do estudo).

Contribuição dos Autores: Concepção e planejamento do estudo: ECD, PSCC, LCR. Coleta, análise e interpretação dos dados: ECD, PSCC, LCR. Elaboração ou revisão do manuscrito: ECD, PSCC, LCR. Aprovação da versão final: ECD, PSCC, LCR. Responsabilidade pública pelo conteúdo do artigo: ECD, PSCC, LCR.

Conflito de Interesses: Os autores declaram não haver conflito de interesses. 